\title{
ASSESSING THE FEASIBILITY OF USING THE RETROGLANDULAR APPROACH IN THE MANAGEMENT OF DEEPLY SEATED MALIGNANT BREAST TUMORS
}

Tarek Abdelhalim El Faiomy, Haitham Mahmoud Fayed, Mahmoud Ahmed Alhussini, Joseph Yousry Youssef

Surgical oncology unit, Department of surgery, Faculty of Medicine, Alexandria University, Alexandria, Egypt

\section{Introduction}

Breast cancer is the most common site-specific cancer among women and it is the leading cause of cancer related deaths among women. It accounts for $29 \%$ of all newly diagnosed cancers in females and is responsible for $14 \%$ of the cancer-related deaths in women. Approximately 1 in 8 women (13\%) will be diagnosed with invasive breast cancer in their lifetime. Understanding the biological behavior and natural history of breast cancer has made breast-conserving therapy (BCT) the choice of treatment in early breast cancer. Oncoplastic breast surgery (OPS) has emerged as a new approach to allow wide excision for BCS without compromising the natural shape of the breast And since one of the main factors for good oncoplastic techniques is the cosmetic outcome,

inframammary crease incision is a good choice as hidden scar in the breast envelope. Here we introduce a new approach for deep breast tumors through an inframammary incision and retro glandular exploration of breast parenchyma which is a simple and easily performed technique

\section{Aim of the work}

The aim of this study was to assess the feasibility of adopting the retro glandular approach for breast conserving surgery by making a skin incision in the inframammary fold (IMF), combined with a retro glandular exploration that leave a hidden scar on the breast skin envelope

\section{Subjects}

After approval of the medical ethics committee of Alexandria Faculty of medicine, an informed consent was taken from all patients to include their data in this study. The study had been conducted on patients presented with deeply seated breast tumors in surgical oncology unit, department of surgery, Alexandria University Hospitals from $01 / 02 / 2020$ to $31 / 1 / 2021$. The selected University Hospitals from $01 / 02 / 2020$ to $31 / 1 / 2021$. The selected
patients had deeply seated breast masses diagnosed as breast patients had deeply seated breast masses diagnosed as breast
cancer by clinical examination ultrasound, mammography and confirmed by ultrasound guided tru cut biopsy.

\section{Methods}

After approval of the medical ethics committee of Alexandria Faculty of medicine an informed consent was taken from all patients to include their data in this study. The tumor outline and the incision are marked with permanent ink while the patient in standing position. The incision is located in the inframammary crease. The entire procedure was carried out with the patient in the supine position with the arm abducted 95 degrees. After incision, dissection was carried out in the plane between the breast parenchyma and pectoralis major muscle. Dissection plane between the breast parenchyma and pectoralis major muscle. Dissection
continues till the mass was identified and an adequate gross safety margin had continues till the mass was identified and an adequate gross safety margin had
been reached. Excision with at least $1 \mathrm{~cm}$ safety margin of normal breast tissue been reached. Excision with at least $1 \mathrm{~cm}$ safety margin of normal breast tissue was done and specimen orientation was made before its delivery outside the
breast. Intraoperative frozen section was done to ensure that adequate microscopic safety margin had been achieved. Axillary lymph node surgery, either axillary clearance or sentinel lymph node biopsy was done through the same incision or separate incision. The defect was closed dir layers.

\section{Exclusion criteria}

Involution of fatty degenerated breast parenchyma.

Unfavorable tumor-to-breast volume ratio.

Previous mastopexy.

Multicenteric breast lesions.

All patients were followed up for the possible early complications and for the cosmetic outcome. The cosmetic outcome of the treated breast was evaluated by breast surgeons in comparison with the untreated breast.

\section{Results}

\begin{tabular}{|c|c|c|c|c|c|c|c|c|}
\hline \multirow{3}{*}{ Cosmetic result } & \multicolumn{6}{|c|}{ Breast size } & \multirow{3}{*}{$\chi^{2}$} & \multirow{3}{*}{${ }^{\mathrm{MC}} \mathbf{p}$} \\
\hline & \multicolumn{2}{|c|}{$\begin{array}{c}\mathbf{A} \\
(n=5) \\
\end{array}$} & \multicolumn{2}{|c|}{$\begin{array}{c}\text { B } \\
(\mathrm{n}=10)\end{array}$} & \multicolumn{2}{|c|}{$\begin{array}{c}C \\
(n=5) \\
\end{array}$} & & \\
\hline & No. & $\%$ & No. & $\%$ & No. & $\%$ & & \\
\hline $\begin{array}{l}\text { Patient satisfaction } \\
\text { Not satisfied }\end{array}$ & 0 & 0.0 & 0 & 0.0 & 2 & 40.0 & & 01 \\
\hline Satisfied & 5 & 100.0 & 10 & 100.0 & 3 & 60.0 & & 0.104 \\
\hline
\end{tabular}

$\chi^{2}$ : Chi square test

\section{Results}

\begin{tabular}{|c|c|c|c|c|c|c|c|c|}
\hline \multirow{3}{*}{ Complications } & \multicolumn{6}{|c|}{ Breast size } & \multirow{3}{*}{$\chi^{2}$} & \multirow{3}{*}{${ }^{\mathrm{MC}} \mathbf{p}$} \\
\hline & \multicolumn{2}{|c|}{$\begin{array}{c}A \\
(n=5)\end{array}$} & \multicolumn{2}{|c|}{$\begin{array}{c}\mathbf{B} \\
(\mathbf{n}=10)\end{array}$} & \multicolumn{2}{|c|}{$\begin{array}{c}\mathrm{C} \\
(\mathrm{n}=5)\end{array}$} & & \\
\hline & No. & $\%$ & No. & $\%$ & No. & $\%$ & & \\
\hline No & 4 & 80.0 & 8 & 80.0 & 1 & 20.0 & & \\
\hline Yes & 1 & 20.0 & 2 & 20.0 & 4 & 80.0 & 5.225 & 0.099 \\
\hline$\overline{\text { Seroma }}$ & 0 & 0.0 & 1 & 10.0 & 3 & 60.0 & & \\
\hline Hematoma & 0 & 0.0 & 0 & 0.0 & 0 & 0.0 & & \\
\hline Wound dehiscence & 1 & 20.0 & 0 & 0.0 & 0 & 0.0 & & \\
\hline Infection & 0 & 0.0 & 1 & 10.0 & 1 & 20.0 & & \\
\hline
\end{tabular}

$\chi^{2}:$ Chi square test, MC: Monte Carlo
p: p value for comparing between the studied categories

Conclusions

Retro glandular approach through an inframammary crease incision is feasible for deeply seated breast cancer. Excellent aesthetic results for small breast cup sizes and acceptable results for larger breast cup sizes had been achieved. This surgical technique had the advantage of leaving no scar on the breast skin envelop, maintaining the natural appearance and shape of the breast and avoiding the need for contralateral symetrization.Patients should be followed for a longer duration to assess the oncologic safety

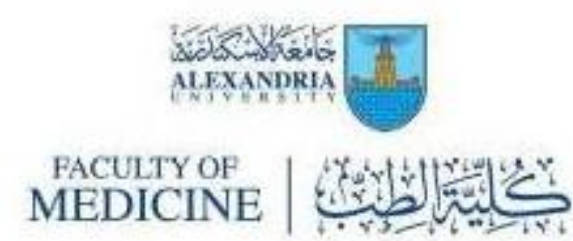

$2021 @$ Alexandria Faculty of Medicine CC-BY-NC

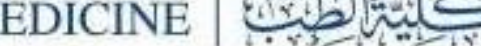

